\title{
Mutation analysis and genomic imbalances of cells found in effusion fluids from patients with ovarian cancer
}

\author{
MARTA BRUNETTI ${ }^{1-3}$, IOANNIS PANAGOPOULOS ${ }^{1}$, ILYÁ KOSTOLOMOV ${ }^{4}$, \\ BEN DAVIDSON $^{2,3}$, SVERRE HEIM $^{1,3}$ and FRANCESCA MICCI ${ }^{1}$ \\ ${ }^{1}$ Section for Cancer Cytogenetics, Institute for Cancer Genetics and Informatics; ${ }^{2}$ Department of Pathology, \\ The Norwegian Radium Hospital, Oslo University Hospital; ${ }^{3}$ Institute of Clinical Medicine, Faculty \\ of Medicine, University of Oslo; ${ }^{4}$ Section for Applied Informatics, Institute for Cancer Genetics and \\ Informatics, The Norwegian Radium Hospital, Oslo University Hospital, 0424 Oslo, Norway
}

Received November 26, 2019; Accepted April 29, 2020

DOI: $10.3892 / 01.2020 .11782$

\begin{abstract}
Ovarian carcinomas and carcinosarcomas often cause malignant effusions, an accumulation within serous cavities of fluid containing cancer cells. Few studies have focused on the molecular alterations and genetic mechanisms behind effusion formation. The present study investigated the mutation status of TP53, PIK3CA, KRAS, HRAS, NRAS and $B R A F$ in effusion fluids from 103 patients with ovarian cancer. In addition, array Comparative Genomic Hybridization $(\mathrm{aCGH})$ analysis was performed on 20 effusions from patients with high-grade serous carcinoma (10 cases positive for TP53 mutation and 10 with TP53 wild-type). TP53 mutations, two of which were novel: c.826_830delCCTGT and c.475_476GC $>\mathrm{TT}$, were identified in $44 \%$ of the cases. Mutations in KRAS, HRAS, and PIK3CA were identified in two, two and four cases, respectively. None of the effusions analysed showed NRAS or BRAF mutations. The aCGH analysis revealed highly imbalanced genomes similar to those described in primary ovarian carcinomas. No specific profile was indicated to distinguish tumors with TP53 mutations from those without. The molecular profiling of cells found in effusion fluids from patients with ovarian cancer thus showed considerable molecular heterogeneity. TP53 seems to be the most frequently mutated gene in these cells and may serve a leading role in the metastatic process.
\end{abstract}

Correspondence to: Dr Francesca Micci, Section for Cancer Cytogenetics, Institute for Cancer Genetics and Informatics, The Norwegian Radium Hospital, Oslo University Hospital, P.O. Box 4954 Nydalen, 0424 Oslo, Norway

E-mail: francesca.micci@labmed.uio.no

Key words: effusions, mutation analysis, high-grade serous ovarian carcinoma, array comparative genomic hybridization, genomic imbalances

\section{Introduction}

Cancers of the ovaries, most of which are carcinomas (OC), are the eighth most common malignancy in women and the most lethal one. In the year 2018, 295,414 new cases were diagnosed and 184,799 deaths occurred from ovarian cancer worldwide (1). OC can be subdivided into various histological subtypes, each showing distinct genomic and epigenomic characteristics (2). High-grade serous carcinoma (HGSC) is the most frequent and aggressive histotype, comprising $70 \%$ of newly diagnosed cases. Less frequent are endometrioid carcinoma (EC, 15\%), clear cell carcinoma (CCC, 12\%), low-grade serous carcinoma (LGSC, <10\%), and mucinous carcinoma (MC, 3\%) (3). Carcinosarcomas (CS) of the female genital tract are biphasic tumors containing some areas showing carcinomatous growth, mostly HGSC, and others displaying sarcomatous differentiation. CS are rare but aggressive tumors that often prove fatal within 1-2 years of diagnosis (4).

The majority of malignant ovarian effusions stem from carcinomas or CS $(5,6)$. They are an almost universal clinical finding in advanced-stage OC, i.e., stage III-IV according to the International Federation of Gynaecology and Obstetrics (FIGO), reflecting widespread intra-abdominal disease with a large number of metastatic tumor cells. OC cells in effusions probably represent a chemoresistant population rendering the disease untreatable and fatal $(7,8)$.

Different cytologic biomarkers are used as adjuncts to morphologic examination to diagnose cancer cells in effusions (5). Studies focusing on molecules that promote the process of invasion and metastasis, as well as influence intracellular signalling pathways and/or act as transcription factors, have provided a better understanding of the biological events behind formation of malignant effusions $(5,8)$; however, this knowledge is still far from complete. Although a growing number of investigations have defined optimal panels for routine cytologic diagnosis of carcinoma cells in effusions, only few studies focused on the molecular alterations and genetic mechanisms behind effusions $(5,9,10)$. And yet, the identification of genetic mutations and genomic imbalances in tumor cells has become increasingly important in the management of different cancer types and also allows us to assess the cells' proneness to develop metastases $(11,12)$. 
We investigated the mutation status of the tumor suppressor gene TP53, the phosphatidylinositol-4,5-bisphosphate 3-kinase catalytic subunit alpha $(P I K 3 C A)$, the protooncogenes of the Ras family-ki-ras2 kirsten rat sarcoma viral oncogene homolog $(K R A S)$, Harvey rat sarcoma viral oncogene homolog (HRAS), the neuroblastoma RAS viral (V-Ras) oncogene homolog (NRAS)-and the v-raf murine sarcoma viral oncogene homolog $(B R A F)$ in a series of 103 ovarian effusions. Furthermore, we performed array comparative genomic hybridization (aCGH) to characterize the genomic imbalances incurred by the cells of 20 effusions from HGSC, of which ten tumors showed TP53 mutations whereas the remaining ten had wild-type TP53.

\section{Materials and methods}

Tumor material. The material consisted of 103 effusions from ovarian cancers, including 84 HGSC, 10 LGSC, two CCC, one EC, and six CS. All patients were treated at The Norwegian Radium Hospital between 2000 and 2015. The diagnoses were reached using a combination of cytological, morphological, and immunohistochemistry (IHC) investigations according to World Health Organization (WHO) 2014 guidelines (3). The study was approved by the Regional Committee for Medical and Health Research Ethics (REK, project number S-04300; http://helseforskning.etikkom.no), the government-appointed committee responsible for overseeing medical ethics in the South-East region of Norway. Informed consent, including consent for publication, was obtained according to national and institutional guidelines. An overview of the cohort used and the clinical and pathological data are given in Table I.

Molecular analyses. DNA was extracted using the Maxwell 16 extractor (Promega) and Maxwell 16 Cell DNA Purification kit (Promega) according to the manufacturer's recommendations. The concentration was measured using QIAxel (Qiagen).

Mutational analysis of TP53, PIK3CA, KRAS, HRAS, and NRAS was performed according to previously described protocols, using M13-linked PCR primers designed to flank and amplify targeted sequences $(13,14)$. The primer combinations BRAF-F1 (5'TGCTTGCTCTGATAGGAAAATGAG ATCT3') and BRAF-R1 (5'ATCTCAGGGCCAAAAATT TAATCAGTG 3') were used to detect the mutation status of $B R A F$. The thermal cycling for $B R A F$ included an initial step at $95^{\circ} \mathrm{C}$ for $10 \mathrm{~min}$ followed by 35 cycles at $96^{\circ} \mathrm{C}$ for $3 \mathrm{sec}$, $58^{\circ} \mathrm{C}$ for $15 \mathrm{sec}, 30 \mathrm{sec}$ at $68^{\circ} \mathrm{C}$, and a final step at $72^{\circ} \mathrm{C}$ for $2 \mathrm{~min}$. Direct sequencing was performed using a $3500 \mathrm{Genetic}$ Analyzer (Applied Biosystems).

The genes were selected based on the information reported in the COSMIC database (Catalogue of Somatic Mutations in Cancer, at https://cancer.sanger.ac.uk/cosmic) (15). According to COSMIC, there is no information on mutations in effusions; however, it contains data on the most frequently mutated genes in ovarian carcinoma. Since KRAS was in the top list, we decided to investigate also the other member genes of the RAS and RAF families, i.e., HRAS, NRAS and BRAF.

The BLAST (http://blast.ncbi.nlm.nih.gov/blast.cgi) and BLAT (http://genome.ucsc.edu/cgi-bin/hgblat) programs were used for computer analysis of sequence data. The reference sequences used for TP53 was NM_000546.5.
Table I. Clinicopathologic parameters of the 103 ovarian effusions investigated.

\begin{tabular}{|c|c|}
\hline Parameter & Distribution (n) \\
\hline \multicolumn{2}{|l|}{ Histology } \\
\hline HGSC & 84 \\
\hline CS & 6 \\
\hline LGSC & 10 \\
\hline $\mathrm{CCC}$ & 2 \\
\hline $\mathrm{EC}$ & 1 \\
\hline \multicolumn{2}{|l|}{ Age } \\
\hline$\leq 60$ & 42 \\
\hline$>60$ & 61 \\
\hline \multicolumn{2}{|l|}{ FIGO stage } \\
\hline I & 1 \\
\hline II & 1 \\
\hline III & 68 \\
\hline IV & 33 \\
\hline \multicolumn{2}{|c|}{ Residual disease } \\
\hline $0 \mathrm{~cm}$ & 23 \\
\hline$\leq 1 \mathrm{~cm}$ & 32 \\
\hline$>1 \mathrm{~cm}$ & 25 \\
\hline $\mathrm{N} / \mathrm{A}$ & 23 \\
\hline \multicolumn{2}{|c|}{ Chemoresponse after primary treatment ${ }^{\mathrm{a}}$} \\
\hline $\mathrm{CR}$ & 53 \\
\hline PR & 32 \\
\hline SD & 7 \\
\hline PD & 1 \\
\hline $\mathrm{N} / \mathrm{A}$ & 10 \\
\hline
\end{tabular}

${ }^{a}$ N/A, Not available (missing data or disease response after chemotherapy could not be evaluated because of normalized CA 125 after primary surgery or missing CA 125 information and no residual tumor). HGSC, high-grade serous carcinoma; CS, carcinosarcoma; LGSC, low-grade serous carcinoma; CCC, clear cell carcinoma; EC, endometrioid carcinoma; N/A, not available; CR, complete response; $\mathrm{PR}$, partial response; $\mathrm{SD}$, stable disease; $\mathrm{PD}$, progressive disease.

The difference between mutation and polymorphism was evaluated by the Genome Aggregation Database (gnomAD; https://gnomad.broadinstitute.org/variant/11-534242-A-G).

Whole genome investigation by means of aCGH was performed using the CytoSure Consortium Cancer + SNP arrays (Oxford Gene Technology) according the manufacturers' recommendation. Data were analysed using Agilent Feature Extraction Software (version 10.7.3.1) and CytoSure Interpret Software (version 4.9.40, Oxford Gene Technology). The genomic imbalances were identified using the Circular Binary Segmentation (CBS) algorithm and adding a custom-made aberration filter defining a copy number aberration (CNA) as a region with minimum five probes gained/lost (16). Annotations are based on human reference sequence GRCh37/hg19.

Twenty samples were selected for aCGH investigation, ten bearing TP53 mutation in their genome and ten wild-type. The 
Table II. Mutation status of TP53.

\begin{tabular}{|c|c|c|c|c|c|}
\hline Case & Histology & TP53 & Case & Histology & TP53 \\
\hline 1 & HGSC & c.437G>A; p.W146* COSM43609 & 54 & HGSC & \\
\hline 2 & HGSC & c.584T>C; p.I195T; COSM11089 & 55 & HGSC & c.475G>C; Pa159P; COSM43836 \\
\hline 3 & HGSC & c. $273 \mathrm{G}>\mathrm{A} ; \mathrm{p} . \mathrm{W} 91^{*} \mathrm{COSM} 44492$ & 56 & HGSC & \\
\hline 4 & HGSC & & 57 & HGSC & \\
\hline 5 & HGSC & c.916C>T; p.R306*; COSM10663 & 58 & HGSC & \\
\hline 6 & HGSC & & 59 & HGSC & \\
\hline $7^{\mathrm{a}}$ & HGSC & c.826_830delCCTGT & 60 & HGSC & c.844C>T; p.R282W; COSM10704 \\
\hline 8 & HGSC & c.818G>A; p.R273H; COSM10660 & 61 & HGSC & c.646G>A; p.V216M; COSM10667 \\
\hline 9 & HGSC & c.797G>A; p.G266E; COSM10867 & 62 & HGSC & c.832 C>T; p.P278S; COSM10939 \\
\hline 10 & HGSC & & 63 & HGSC & \\
\hline 11 & HGSC & c.488A>G; p.Y163C; COSM10808 & 64 & HGSC & \\
\hline 12 & HGSC & c.524G>A; p.R175H; COSM10648 & 65 & HGSC & \\
\hline 13 & HGSC & c.844C>T; p.R282W; COSM10704 & 66 & HGSC & \\
\hline 14 & HGSC & c.574C>T; p.Q192*; COSM10733 & 67 & HGSC & \\
\hline 15 & HGSC & c.527G>T; p.C176F; COSM10645 & 68 & HGSC & \\
\hline 16 & HGSC & c.469G >T; p.V157F; COSM10670 & 69 & HGSC & \\
\hline 17 & HGSC & c.527G>A; p.C176Y; COSM10687 & 70 & HGSC & \\
\hline 18 & HGSC & & 71 & HGSC & c.527G>T; p.C176F; COSM10645 \\
\hline 19 & HGSC & c.754del; p. L252fs"93; COSM45215 & 72 & HGSC & \\
\hline 20 & HGSC & c.403del; p.C135fs*35; COSM44670 & 73 & HGSC & \\
\hline 21 & HGSC & & 74 & HGSC & \\
\hline 22 & HGSC & c. $394 \mathrm{~A}>\mathrm{T} ;$ p.K132*; COSM44641 & 75 & HGSC & c.578A>G; p.H193R; COSM10742 \\
\hline 23 & HGSC & c.832C>G; p.P278A; COSM10814 & 76 & HGSC & \\
\hline 24 & HGSC & c.814G>A; p.V272M; COSM10891 & 77 & HGSC & \\
\hline 25 & HGSC & c.394A>G; p.K132E; COSM10813 & 78 & HGSC & \\
\hline $26^{\mathrm{a}}$ & HGSC & c. $475 \_476 \mathrm{GC}>\mathrm{TT}$ & 79 & HGSC & \\
\hline 27 & HGSC & & 80 & HGSC & \\
\hline 28 & HGSC & c.797G>A; p.G266E; COSM10867 & 81 & HGSC & c.796G>A; p.G266R; COSM10794 \\
\hline 29 & HGSC & c.108G>A; p.P36P; COSM6474191 & 82 & HGSC & c.844C>T; p.R282W; COSM10704 \\
\hline & & c.737T>A; p.M246K; COSM44103 & 83 & HGSC & \\
\hline 30 & HGSC & c.742C>T; p.R248W; COSM10656 & 84 & HGSC & \\
\hline 31 & HGSC & & 85 & LGSC & c.750del; p. I251fs"94; COSM44064 \\
\hline 32 & HGSC & c.488A>G; p.Y163C; COSM10808 & 86 & LGSC & \\
\hline 33 & HGSC & c.836G>A; p.G279E; COSM43714 & 87 & LGSC & c.714T>A; p.C238*; COSM45677 \\
\hline 34 & HGSC & & 88 & LGSC & \\
\hline 35 & HGSC & c.818G>A; p.R273H; COSM10660 & 89 & LGSC & \\
\hline 36 & HGSC & & 90 & LGSC & \\
\hline 37 & HGSC & & 91 & LGSC & \\
\hline 38 & HGSC & & 92 & LGSC & \\
\hline 39 & HGSC & c.524G>A; p.R175H; COSM10648 & 93 & LGSC & \\
\hline 40 & HGSC & & 94 & LGSC & \\
\hline 41 & HGSC & c.711G>A; p.M237I; COSM10834 & 95 & $\mathrm{CCC}$ & \\
\hline 42 & HGSC & & 96 & $\mathrm{CCC}$ & \\
\hline 43 & HGSC & c.166G>T; p.E56*; COSM12168 & 97 & $\mathrm{EC}$ & c.1024C>T; p.R342*; COSM11073 \\
\hline 44 & HGSC & c.524G>A; p.R175H; COSM10648 & 98 & CS & c.796G>A; p.G266R; COSM10794 \\
\hline 45 & HGSC & & 99 & $\mathrm{CS}$ & \\
\hline 46 & HGSC & & 100 & $\mathrm{CS}$ & \\
\hline 47 & HGSC & & 101 & CS & \\
\hline 48 & HGSC & & 102 & $\mathrm{CS}$ & \\
\hline 49 & HGSC & & 103 & CS & \\
\hline 50 & HGSC & & & & \\
\hline 51 & HGSC & & \multirow{3}{*}{\multicolumn{3}{|c|}{$\begin{array}{l}\text { aNovel mutation site; }{ }^{*} \text {, stop codon; HGSC, high-grade serous carci- } \\
\text { noma; CS, carcinosarcoma; LGSC, low-grade serous carcinoma; } \\
\text { CCC, clear cell carcinoma; EC, endometrioid carcinoma. }\end{array}$}} \\
\hline 52 & HGSC & c.434T>C; p.L145P; COSM43899 & & & \\
\hline 53 & HGSC & & & & \\
\hline
\end{tabular}

Table II. Continued.

${ }^{\text {aNovel mutation site; }}$, , stop codon; HGSC, high-grade serous carci$\mathrm{CCC}$, clear cell carcinoma; EC, endometrioid carcinoma. 


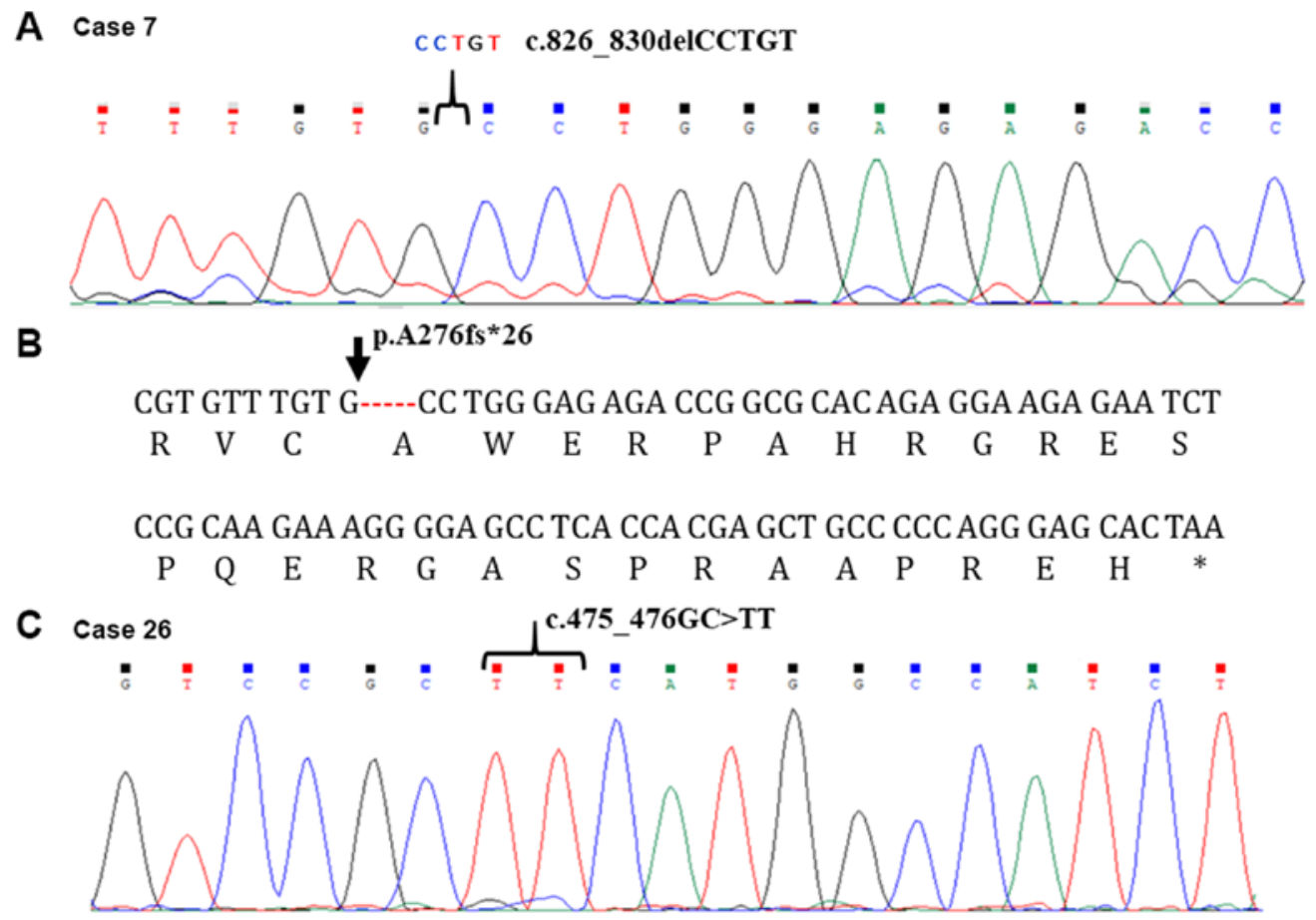

Figure 1. Novel site mutations for TP53 (A) Partial sequence chromatogram of case 7 showing delCCTGT; (B) open reading frame of case 7; (C) partial sequence chromatogram of case 26 showing the substitution GC>TT.

average copy number alteration (ANCA) index was calculated as the total number of aberrations divided by the samples number between the two groups (17). The statistical analysis was performed using the Mann-Whitney U test.

\section{Results}

All effusions analyzed for TP53, PIK3CA, KRAS, HRAS, $N R A S$, and $B R A F$ mutation status gave informative results. TP53 was found mutated in 41 out of 84 HGSC (49\%), in two out of 10 LGSC (20\%), in the only case of EC examined, and in one out of six CS. A detailed overview of the TP53 findings is shown in Table II. Two novel mutation sites were identified for TP53: c.826_830delCCTGT in case 7 and c.475-476GC $>$ TT in case 26 (Fig. 1). PIK3CA mutations were found in four HGSC of 103, in which a c.1634A $>\mathrm{C}$ (cases 2,56, and 58) and a c. $3155 \mathrm{C}>\mathrm{T}$ mutation (case 79 ) were seen. We identified the c. $34 \mathrm{G}>\mathrm{T}$ and c.183A $>\mathrm{C}$ KRAS mutations in two of 103 specimens (cases 10, a HGSC, and 85, an LGSC, respectively). The HRAS mutation c. $173 \mathrm{C}>\mathrm{T}$ was also detected in two tumors (2\%; cases 16 and 23), both of them HGSC. Finally, we identified an $H R A S$ polymorphism, c.81T $>\mathrm{C}$, in 38 effusions (37.5\%) of all histotypes. None of the tumors showed a mutated sequence for $N R A S$ or $B R A F$.

aCGH analysis for genomic imbalances was performed on 20 effusions from patients with HGSC, comparing 10 tumors bearing TP53 mutations (cases 1, 3, 5, 7, 8, 13, 14, 15, 19, and 32) and 10 which had wild-type TP53 sequence (cases 18 , $27,31,36,37,38,42,45,47$, and 48). Overall, the aCGH analysis revealed highly imbalanced genomes in all tumors analysed with many gains and/or losses (Table SI). The most frequent gains were scored at $8 \mathrm{q} 24.3,20 \mathrm{q} 13.2$, and $20 \mathrm{q} 13.31$ (70\%) whereas the most frequent losses were scored at $4 \mathrm{q} 25$ and 4q26 (75\%) (Fig. 2). Amplifications mostly involved chromosomal band $19 \mathrm{q} 11$ followed by the segment $3 \mathrm{q} 22 \mathrm{q} 29$. The two subgroups of effusions, i.e., with and without TP53 mutation, were both very complex and similar with regard to imbalances. The ANCA index calculated for tumors (18) with TP53 mutation was 83.2 but 66.3 for tumors with wild-type TP53 (P=0.14).

\section{Discussion}

Molecular profiles of different tumor types have helped manage cancer patients with regard to diagnosis, prognosis, and lately also choice of treatment (19). A similar molecular characterization of effusions from ovarian cancer might highlight the mechanisms behind development of metastasis and possibly, further down the road, help decide among different personalized therapies (5). Since the number of studies focusing on molecular analysis of ovarian cancers at such advanced stage that effusions have already developed, is low, and since chemoresistance is one of the main characteristics of these malignancies, we aimed to add to the existing knowledge by performing mutation analyses of selected genes as well as determining copy number profiles of two groups of patients, those whose tumors did or did not have TP53 mutations.

The tumor suppressor gene TP53 has been found mutated in many different malignancies (20), including those arising in the ovaries, at a frequency of $66 \%$ in the most aggressive serous carcinomas (21). The rate of TP53 mutation detected in our series was $46 \%$ for effusions from HGSC and LGSC. The seeming discrepancy between the frequencies recorded in the present series and in the literature could be due to methodological limitations, see below. In HGSC, we identified 

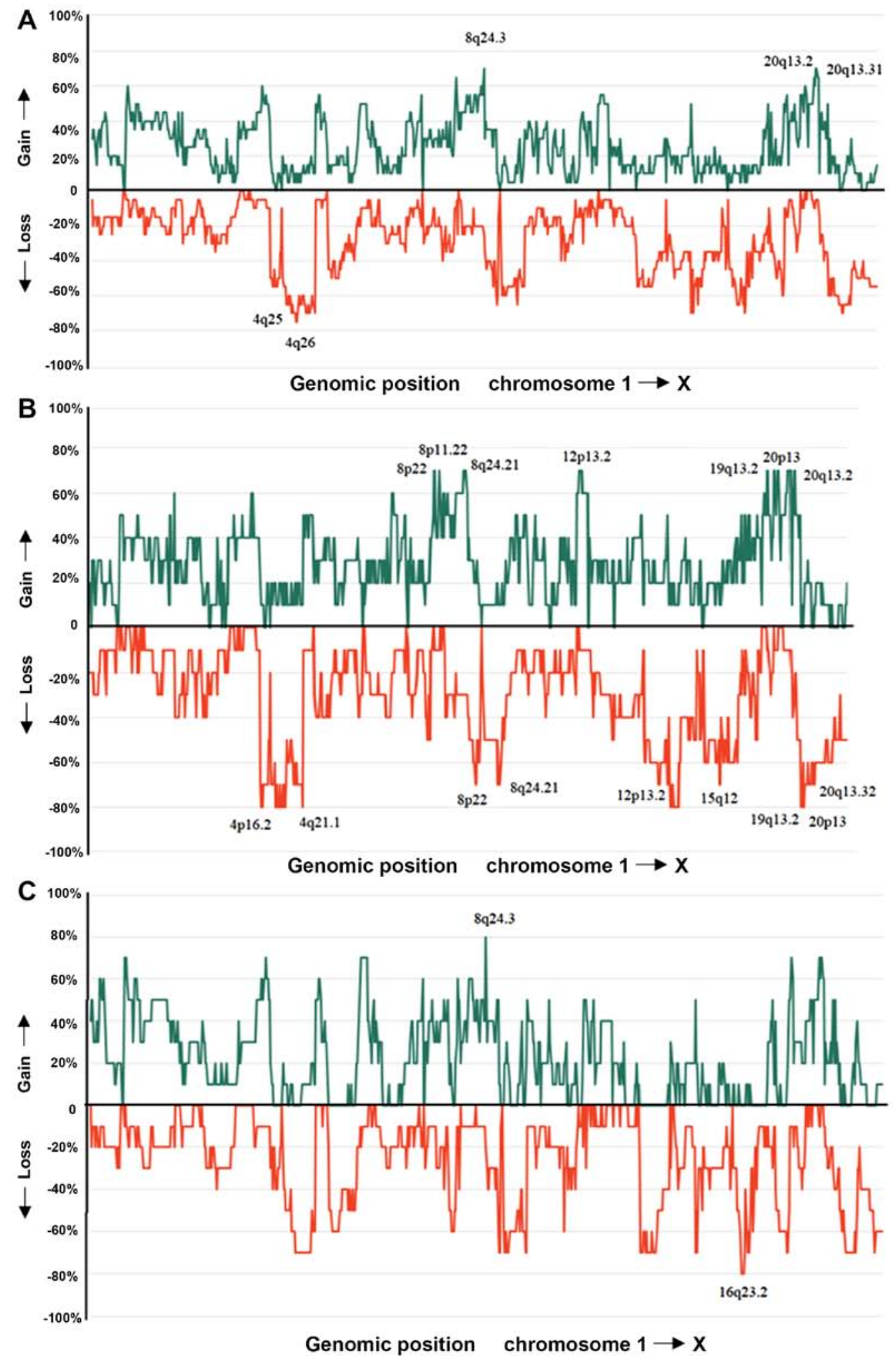

Figure 2. Profiles of imbalances detected by aCGH. (A) Gains and losses detected in effusion cells in patients with HGSC whose tumors were either TP53 mutated or wild-type; (B) Genomic gains and losses in TP53 mutated HGSC effusions; (C) Genomic gains and losses in TP53 wild-type HGSC effusions. aCGH, array Comparative Genomic Hybridization; HGSC, high-grade serous carcinoma.

two novel sites for TP53 mutation: A deletion of the CCTGT sequence was found in position c.826_830 of case 7 (stage III tumor), whereas a substitution GC>TT in position 475_476GC was identified in case 26 (stage IV tumor). The c.826_830del CCTGT is an out-of-frame change resulting in a frameshift of 26 amino acids (aa) (p. A276fs*26) (Fig. 1) after which a stop codon occurs. The predicted protein would consist of 156 aa. The substitution c.475_476GC $>$ TT results in a change from alanine (A) to phenylalanine (F) (p.A159F). The mutation is at present of unknown pathogenicity in ovarian cancer. However, other mutations on c. 475 have been reported as pathogenic in the COSMIC database, e.g., in tumors of the lung and liver 
(https://cancer.sanger.ac.uk/cosmic). The impact of the new mutation sites in relation to different clinical parameters awaits further studies, ideally of larger series of patients. The two patients here examined had received upfront surgery and standard chemotherapy; case 7 showed a residual disease of $6 \mathrm{~cm}$ whereas case 26 had no residual disease at primary operation. Furthermore, both cases showed relatively long survival: Case 7 had 13 months progression-free survival (PFS) and overall survival (OS) of 81 months, whereas case 26 had PFS of 27 months and OS of 45 months.

PIK3CA belongs to the family of genes encoding phosphatidylinositol 3-kinases (PI3Ks). It is activated through the PI3K/AKT signalling pathway in $70 \%$ of ovarian cancers, promoting cellular growth, proliferation, and cell survival (22). Somatic mutations of this gene have been detected in different cancer types (23). In ovarian cancer, it occurs in $30 \%$ of all tumors, but reaches $45 \%$ in EC and CCC (24). We found PIK3CA mutated in $4 \%$ of the HGSC effusions examined, which is in line with what is reported in the COSMIC database. Unfortunately, the number of EC and CCC samples was too low to allow statistical conclusions. A number of clinical studies have focused on the PI3K/AKT/mTOR signaling pathway as a therapeutic target for patients with ovarian cancer $(25,26)$; the identification of patients carrying $P I K 3 C A$ mutation may therefore be important for the choice of therapy. Important to note in this regard is the fact that also other genes of the PI3K/AKT/mTOR signaling pathway should be investigated for their mutation status as they, too, may be involved pathogenetically (26).

$K R A S$ and $H R A S$ are principal members of the RAS family and have frequently been implicated in the development of different types of tumors (27). In ovarian carcinomas, the incidence of $K R A S$ point mutations was found to be $13 \%$ (21). Previous studies have demonstrated an association between $K R A S$ mutations and well-differentiated, clinically less advanced cancers $(28,29)$. KRAS mutation was in ovarian serous carcinoma found more frequently in LGSC than in HGSC (30-32).

HRAS mutations are rare in ovarian tumors $(33,34)$. We found an HRAS mutation in only two HGSC: However, our study showed presence of the $81 \mathrm{~T}>\mathrm{C}$ polymorphism in the coding region of HRAS in 38 out of 103 tumors (37\%) of all histotypes. The Genome Aggregation Database, gnomAD, reports that SNP $81 T>C$ is a polymorphism seen in $30 \%$ of the normal population. Both tumors with HRAS mutation also showed TP53 mutation. In each case, one can hypothesize a scenario in which the mutations represent a primary and a secondary event either in the same cell or in different cells/clones.

Information on effusions from CS arising in the female genital tract is limited to data generated by immunohistochemical techniques (35). This is the first time that mutation analyses have been performed on such metastatic cells. It seems, however, that the genes investigated in the present study are not relevant in cells from effusions since we found only one CS with TP53 mutation.

The mutation rates for the analysed genes in the present study differ slightly from those reported in the literature, something that may be attributable to the molecular methods applied. We used PCR followed by Sanger sequencing. It is known that Sanger sequencing cannot detect mutation if the level of abnormal cells is below $15 \%$ (36), whereas next generation sequencing (NGS) or exome sequencing, used in most published studies (37), is more sensitive, i.e., has a higher resolution level. NGS, on the other hand, cannot discriminate between a 'real' mutation and a polymorphism. Taking into account these two factors, one would indeed expect higher mutation rates to be detected by NGS compared to Sanger sequencing, as was observed.

aCGH data showed highly imbalanced genomes both in tumors with mutated and wild-type TP53. The genomic regions involved are in agreement with the results of previous studies where primary OC were investigated (38). The ANCA index detected in the TP53 mutated subgroup was 83.2 whereas it was 66.3 in the subgroup with wild-type TP53. The difference between the two groups was not found statistically significant using the Mann-Whitney U test.

The origin of ovarian carcinomas has lately been debated but, according to the latest WHO classification, the majority of HGSC are thought to originate in the tubes whereupon metastatic spreading occurs to the ovaries $(39,40)$. In the light of this concept, it is not surprising that ovarian carcinomas show the same imbalances as do ovarian cancer cells found in effusions, since both represent late evolutionary stages in carcinoma development.

\section{Acknowledgements}

The authors wish to thank Miss Margrethe Stoltenberg and Dr Rønnaug A. U. Strandabø, both from the Section for Cancer Cytogenetics, Institute for Cancer Genetics and Informatics, Oslo University Hospital, for technical assistance.

\section{Funding}

This work was supported by grants from the South-East Norway Regional Health Authority (Helse Sør-Øst) and Radiumhospitalets Legater.

\section{Availability of data and materials}

The datasets used and/or analyzed during the current study are available from the corresponding author on reasonable request.

\section{Authors' contributions}

MB performed molecular experiments and wrote the manuscript. IP participated in performing molecular experiments and interpretation of data. IK participated in performing data analysis. BD provided clinical data and specimens. SH assisted with writing of the article and experimental design. FM designed the study and supervised the writing of the manuscript. All authors have read and approved the final version of the manuscript.

\section{Ethics approval and consent to participate}

The ethical approval was granted by the Regional Committee for Medical and Health Research Ethics (REK; http://helseforskning.etikkom.no); for further information, please see this website: http://www.eurecnet.org/information/ norway.html. 


\section{Patient consent for publication}

Consent for publication of data was provided by all patients.

\section{Competing interests}

The authors declare that they have no competing interests.

\section{References}

1. Bray F, Ferlay J, Soerjomataram I, Siegel RL, Torre LA and Jemal A: Global cancer statistics 2018: GLOBOCAN estimates of incidence and mortality worldwide for 36 cancers in 185 countries. CA Cancer J Clin 68: 394-424, 2018.

2. Prat J, D'Angelo E and Espinosa I: Ovarian carcinomas: At least five different diseases with distinct histological features and molecular genetics. Hum Pathol 80: 11-27, 2018.

3. Kurman RJ, Carcangiu ML, Herrington CS and Young RH: WHO classification of tumors of female reproductive organs. IARC, 2014.

4. D'Angelo E and Prat J: Pathology of mixed Mullerian tumours. Best Pract Res Clin Obstet Gynaecol 25: 705-718, 2011.

5. Davidson, B: Ovarian and primary peritoneal carcinoma. In: Serous Effusions - Etiology, Diagnosis, Prognosis and Therapy. Davidson B, Firat P, Michael CW (eds). Springer, London, UK, pp47-68, 167-204, 2011.

6. Piche A: Malignant peritoneal effusion acting as a tumor environment in ovarian cancer progression: Impact and significance. World J Clin Oncol 9: 167-171, 2018.

7. Davidson B: Recently identified drug resistance biomarkers in ovarian cancer. Expert Rev Mol Diagn 16: 569-578, 2016.

8. Davidson B: Biomarkers of drug resistance in ovarian cancer-an update. Expert Rev Mol Diagn 19: 469-476, 2019.

9. Brunetti M, Holth A, Panagopoulos I, Staff AC, Micci F and Davidson B: Expression and clinical role of the dipeptidy peptidases DPP8 and DPP9 in ovarian carcinoma. Virchows Archiv 474: 177-185, 2019.

10. Davidson B, Stavnes HT, Holth A, Chen X, Yang Y, Shih IeM and Wang TL: Gene expression signatures differentiate ovarian/peritoneal serous carcinoma from breast carcinoma in effusions. J Cell Mol Med 15: 535-544, 2011.

11. Shah RH, Scott SN, Brannon AR, Levine DA, Lin O and Berger MF: Comprehensive mutation profiling by next-generation sequencing of effusion fluids from patients with high-grade serous ovarian carcinoma. Cancer Cytopathol 123: 289-297, 2015.

12. Nagel H, Schulten HJ, Gunawan B, Brinck U and Fuzesi L: The potential value of comparative genomic hybridization analysis in effusion-and fine needle aspiration cytology. Mod Pathol 15: $818-825,2002$.

13. Malcikova J, Tausch E, Rossi D, Sutton LA, Soussi T, Zenz T, Kater AP, Niemann CU, Gonzalez D, Davi F, et al: ERIC recommendations for TP53 mutation analysis in chronic lymphocytic leukemia-update on methodological approaches and results interpretation. Leukemia 32: 1070-1080, 2018.

14. Brunetti M, Agostini A, Staurseth J, Davidson B, Heim S and Micci F: Molecular characterization of carcinosarcomas arising in the uterus and ovaries. Oncotarget 10: 3614-3624, 2019.

15. Tate JG, Bamford S, Jubb HC, Sondka Z, Beare DM, Bindal N, Boutselakis H, Cole CG, Creatore C, Dawson E, et al: COSMIC: The catalogue of somatic mutations in cancer. Nucleic Acids Res 47: D941-D947, 2019.

16. Olshen AB, Venkatraman ES, Lucito R and Wigler M: Circular binary segmentation for the analysis of array-based DNA copy number data. Biostatistics 5: 557-572, 2004.

17. Ried T, Heselmeyer-Haddad K, Blegen H, Schröck E and Auer G Genomic changes defining the genesis, progression, and malignancy potential in solid human tumors: A phenotype/genotype correlation. Genes Chromosomes Cancer 25: 195-204, 1999.

18. Micci F, Teixeira MR, Haugom L, Kristensen G, Abeler VM and Heim S: Genomic aberrations in carcinomas of the uterine corpus. Genes Chromosomes Cancer 40: 229-246, 2004.

19. Jackson SE and Chester JD: Personalised cancer medicine. Int J Cancer 137: 262-266, 2015.
20. Zhang W, Edwards A, Flemington EK and Zhang K: Significant prognostic features and patterns of somatic TP53 mutations in human cancers. Cancer Inform 16: 1176935117691267, 2017.

21. www.sanger.ac.uk/genetics/CGP/cosmic.

22. Li H, Zeng J and Shen K: PI3K/AKT/mTOR signaling pathway as a therapeutic target for ovarian cancer. Arch Gynecol Obstet 290: 1067-1078, 2014.

23. Samuels Y and Waldman T: Oncogenic mutations of PIK3CA in human cancers. Curr Top Microbiol Immunol 347: 21-41, 2010.

24. Campbell IG, Russell SE, Choong DY, Montgomery KG, Ciavarella ML, Hooi CS, Cristiano BE, Pearson RB and Phillips WA: Mutation of the PIK3CA gene in ovarian and breast cancer. Cancer Res 64: 7678-7681, 2004.

25. Mabuchi S, Kuroda H, Takahashi R and Sasano T: The $\mathrm{PI} 3 \mathrm{~K} / \mathrm{AKT} / \mathrm{mTOR}$ pathway as a therapeutic target in ovarian cancer. Gynecol Oncol 137: 173-179, 2015.

26. Gasparri ML, Bardhi E, Ruscito I, Papadia A, Farooqi AA, Marchetti C, Bogani G, Ceccacci I, Mueller MD and Benedetti Panici P: PI3K/AKT/mTOR pathway in ovarian cancer treatment: Are we on the right track? Geburtshilfe Frauenheilkd 77: 1095-1103, 2017.

27. Fernández-Medarde A and Santos E: Ras in cancer and developmental diseases. Genes Cancer 2: 344-358, 2011.

28. Nodin B, Zendehrokh N, Sundstrom M and Jirstrom K: Clinicopathological correlates and prognostic significance of KRAS mutation status in a pooled prospective cohort of epithelial ovarian cancer. Diagn Pathol 8: 106, 2013.

29. Dobrzycka B, Terlikowski SJ, Kowalczuk O, Niklińska W, Chyczewski L and Kulikowski M: Mutations in the KRAS gene in ovarian tumors. Folia Histochem Cytobiol 47: 221-224, 2009.

30. Della Pepa C, Tonini G, Santini D, Losito S, Pisano C, Di Napoli M, Cecere SC, Gargiulo P and Pignata S: Low grade serous ovarian carcinoma: From the molecular characterization to the best therapeutic strategy. Cancer Treat Rev 41: 136-143, 2015.

31. Singer G, Shih Ie M, Truskinovsky A, Umudum $H$ and Kurman RJ: Mutational analysis of K-ras segregates ovarian serous carcinomas into two types: Invasive MPSC (low-grade tumor) and conventional serous carcinoma (high-grade tumor). Int J Gynecol Pathol 22: 37-41, 2003.

32. Singer G, Oldt R III, Cohen Y, Wang BG, Sidransky D, Kurman RJ and Shih IeM: Mutations in BRAF and KRAS characterize the development of low-grade ovarian serous carcinoma. J Natl Cancer Inst 95: 484-486, 2003.

33. Hunter SM, Anglesio MS, Ryland GL, Sharma R, Chiew YE, Rowley SM, Doyle MA, Li J, Gilks CB, Moss P, et al: Molecular profiling of low grade serous ovarian tumours identifies novel candidate driver genes. Oncotarget 6: 37663-37677, 2015.

34. Hollis RL and Gourley C: Genetic and molecular changes in ovarian cancer. Cancer Biol Med 13: 236-247, 2016.

35. Ikeda K, Tate G, Suzuki T and Mitsuya T: Effusion cytodiagnosis of carcinosarcoma derived from the female genital tract: Immunohistochemical features of MMP-7 and Ki-67 and immunofluorescence double staining analyses of eight cases. Gynecol Oncol 97: 323-329, 2005.

36. Rohlin A, Wernersson J, Engwall Y, Wiklund L, Bjork J and Nordling M: Parallel sequencing used in detection of mosaic mutations: Comparison with four diagnostic DNA screening techniques. Hum Mutat 30: 1012-1020, 2009.

37. Salk JJ, Schmitt MW and Loeb LA: Enhancing the accuracy of next-generation sequencing for detecting rare and subclonal mutations. Nat Rev Genet 19: 269-285, 2018.

38. Micci F, Haugom L, Abeler VM, Davidson B, Trope CG and Heim S: Genomic profile of ovarian carcinomas. BMC cancer 14: 315, 2014

39. Kim J, Park EY, Kim O, Schilder JM, Coffey DM, Cho CH and Bast RC Jr: Cell origins of high-grade serous ovarian cancer. Cancers (Basel) 10: 433, 2018.

40. Lengyel E: Ovarian cancer development and metastasis. Am J Pathol 177: 1053-1064, 2010.

This work is licensed under a Creative Commons Attribution-NonCommercial-NoDerivatives 4.0 International (CC BY-NC-ND 4.0) License. 